\title{
Der Streit um das Ding an sich und seine Frneuerung im sozialistischen Lager. \\ Von Franz Staudinger.
}

Ein altes Gespenst geht wieder um, das man schon zuweilen gebannt glaubte. Das „Ding an sich", das jenseits der Erscheinung unerkennbar za thronen pflegt, tritt wieder, gleich der weissen Fran im Hohenzollernschloss, in Erscheinung. Und diese Erscheinung des Dinges an sich findet heute statt in sozialistischen, bezw. marxistischen Kreisen. Ob Heil oder Unheil verkündend, das muss die Zukunft lehren; vorläntig hat es nur Streit erregt.

In diesem Streite stand auf der einen Seite der strenge Materialist alter Observanz Ge org Ple chanow, bekannt durch seine „Beiträge zar Geschichte des Materialismus", worin er scharfe Ausfälle gegen die Kantianer, besonders gegen F. A. Lange gemacht hat, und auf der anderen Seite standen Eduard Bernstein sowie Dr. Konrad Schmidt, die bei Kant eine Vertiefung ihrer Philosophie zu finden hoffen. ${ }^{1}$ )

Das Problem, das hier in Frage steht, ist von Plechanow dahin entwickelt worden, dass Kant sich widerspreche, indem er einerseits richtig die Dinge an sich als Ursache unserer Empfindungen ansehe, andererseits aber behaupte, dass die Kategorie der Kausalität

1) Die einschlägigen Arbeiten sind:

Georg Pleohanow: Beiträge zur Geschichte des Materialismus. Stuttgart. 1896.

Konrad Schmidt: Kritik zu vorgenanntem Buch im „Sozialistischen Akademiker". 1896. Juli- und Angustheft.

K. Schmidt: Über Krouenbergs Buch: Kant, sein Leben und seine Lehre. Torwärts, 17. Okt. 1897. 8. Beil.

Eduard Bernstein: Das realistische und ideologische Element im Sozialismus: „Die Neue Zeit", Stattgart 1897/98. Heft 34 u. 39.

G. Plechanow: Bernstein und der Materialismus. Ébenda: Heft 44.

G. Plechanow: Konrad Schmidt gegen Karl Marz und Friedr. Engels Ebenda, 1898/99. Heft 5 .

K. Schmidt: Einige Bemerkungen uber Plechanow. Ebenda No. 11.

G. Plechanow: Materialismus oder Kantianismus. Ebenda, No. 19 u. 20.

Dr. Ch. Schitlowsky: Die Polemik Pleobanow contra Stern und Konrad Schmidt. "Sozialistische Monatshefte", 1899, Juni u. Juli. 
auf die Dinge an sich nicht anwendbar sei. ${ }^{1}$ ) Konrad Schmidt dagegen, dem Bernstein den Austrag des Streits zuschiebt, vertritt den Gedanken, dass Kants Lebre wesentlich eine Theorie der Erfahrung sei, innerbalb deren uberall streng kausale Verknupfung der Erscheinungen stattfinde. Dieser Gedanke, den man auch Materialismus nennen könnte, sei aber grundverschieden von dem metaphysischen Materialismus, der „die Elemente der Erscheinungen fur Dinge an sich erklärt." 2 )

Es ist nicht zweifelhaft, dass hier beide Streiter aneinander rorbeireden. Was Plechanow betont wissen will, lässt Konrad Schmidt völlig kalt, und umgekehrt kennt Plechanow nicht die Probleme, welche Kant zu der Trennung des Dings an sich von den Erscheinungen geführt haben und notwendig flibren mussten. Er bänmt sich mit der Vebemenz and den Waffen des common sense gegen eine Schlussfolgerung, deren Prämissen er nicht erfasst hat. Darum kann er nattirlich auch bei seinem Gegner auf kein Verstïndnis desjenigen Einwands hoffen, der ihm am Herzen liegt, und den wir allerdings als sehr berechtigt, wenn auch als falsch gefasst, erkennen mulssen.

So steht der Sachrerhalt; - aber nicht etwa bloss in sozialistischen Kreisen. Der zwischen genannten Sozialisten ausgebrochene Streit spiegelt nur den Zustand wieder, in dem sich das Erkenntnisproblem noch hente tiberhaupt befindet. Die einen, anf dem Boden der Empirie and Psychologie stehend, verkennen die erkenntnistheoretische Hauptleistang Kants, die anderen, zam Verständnis dieser Errangenschaft vorgedrangen, vergessen dartuber, eine empfindliche Licke zu beachten, die Kant nicht nur offen gelassen hat, was nichts geschadet hätte, sondern die er durch eịne widersprachsvolle Annahme zu schliessen suchte. Infolge dieses Lösungsversuchs von Kant selbst muss das „Ding an sich" immer wieder als Gespenst erscheinen and Verwirrang stiften.

Wir wollen den Versuch machen, diesen armen, irren Geist zar Rahe im Orkas za bringen. Dazn ist es nötig, dass wir uns karz die wesentlichsten Erkenntnisfragen vor Angen fubren, die bis zu Kant die Denker beschäftigt haben, sodann die wertvolle Entdeckang Kants skizzieren, drittens das Problem offen legen, das anch hiernach noch. zu lösen bleibt, and endlich den Weg, der za dessen Lösung fubren möchte, andeuten.

1) N. Z. 1898/99, Heft 5, S. 136.

2) N. Z. a. a. 0., No. 11, S. 326 . 
Das geschichtlich erste Problem, welches die Philosophie beschäftigt hat, ist die Frage: Was ist das eigentliche Sein in dem Wechsel der Erscheinungen. Dem zam Selbstbewasstsein erwachenden Denken wohnt ja der Drang inne, in dem Vielen and Wechselnden Einheit zu suchen. Und diese Einheit fand z. B. ein Thales im Wasser, ein Anaximenes im Apeiron, ein Parmenides in der den Raum erfullenden einheitlichen und ewigen Kugel etc. etc. Diese Nachforschung nach der sachlichen Grundlage des Seins wird heute, mit Ausnahme von einigen Metaphysikern, nicht mehr von den Philosophen betrieben. Die Naturwissenschaft, insbesondere Physik und Chemie, sind an deren Stelle getreten und forschen anf Grand ihrer allmäblich immer sichereren Methoden nach dem einheitlichen Zusammenhange dessen, was ist.

Allein indem man jene Forschung nach dem Seienden betrieb, musste natargemäss eine zweite Frage auftreten: Wodurch erkennen wir? Welches sind die Erkenntnismittel, durch die wir dem Seienden beizukommen, es in unsere Erkenntnis aufzunehmen vermögen? Diese Frage liegt schon der Eleatischen Philosophie zu Grunde. Wenn Parmenides das als ewige Kugel vorhandne Seiende für das Denkbare and das Denkbare für wirklich erklärt, dem wechselnden Sinnenschein aber keine Wahrheit zugesteht, so hat er bereits den Schritt über die blosse Erforschang des Seins hinaus gethan und Erkenntniskritik geubt. Er hat die Erkenntnismittel bereits in Sinneswahrnehmungen und Gedanken getrennt, and unter Abweisung der ersteren für das Denken als allein adäquates Erkenntnismittel Partei ergriffen. Und umgekehrt liegt, wenn auch minder deutlich, der Heraklitischen Lehre vom ewigen Flusse der Gedanke von der massgebenden Bedeutang der den ewigen Wechsel vor Augen führenden Sinneswahrnehmungen zu Grande, ein Gedanke, der bei Protagoras and seinen Schülern an die Spitze getrieben wird. Die Objektivität der Dinge geht hier in der Subjektivität der Erscheinungen auf. Unsere modernen "Positivisten" kommen so ziemlich za dieser Anschanung zuriick.

Auf diesem Boden der ständigen Vermengang ron Erforschang des Seienden and Kritik der Erkenntnismittel bewegt sich die Philosophie bis auf Kant. Als charakteristisch in dieser Denkentwicklang sei die Aufstellang Lockes herrorgehoben. Ohne weiteres gleitet dieser von der Trennung der Erkenntnismittel in Sensation and Reflexion hinuber za dem Unterschiede der sekundären Eigenschaften der Dinge, der Töne, Farben etc., 
die keinem existierenden Dinge ähnlich sind, and der primären Eigenschaften, Grösse, Zabl, Gestalt, die in den Körpern selber wahrgenommen werden. Eine kritische Untersuchnng der B6fagnis der Erkenntnismittel zu solchen Feststellungen fehlt.

Ein wirklicher Fortschritt war erst möglich, als man lernte, grundsätzlich za fragen, ob nicht selbst diejenigen Begriffe, die man ohne weiteres za wirklichen oder scheinbaren Eigenschaften der Dinge gemacht hatte, zanächst einmal selbst als Erkenntnismittel za betrachten, und auf ibre Bedeatang in dieser Hinsicht za untersuchen seien. Denn alle Vorstellangen, durch welche wir Objekte erkennen, sind, sofern sie das leisten, Mittel zar Erkenntnis.

Diese, bekanntlich anf David Humes Forschungen rahende Problemstellang and sodann die Feststellung der zar Konstraktion der Erfahrang notwendigen Erkenntnis. mittel ist die grundlegende Leistang Kants. Darin allein besteht die Kopernikanische Bedeutung seiner Lehre. Er selbst stellt diese freilich in der Vorrede zar zweiten Auflage der Kritik anders dar: „Wenn die Anschaunng [oder der Begriff] sich nach der Beschaffenheit der Gegenstände richten müsste, so sehe ich nicht ein, wie man a priori davon etwas wissen könne; richtet sich aber der Gegenstand nach der Beschaffenheit unseres Anschanungsvermögens, [bezw. nach den Regeln des Verstandes, die ich „in mir, noch ehe mir Gegenstände gegeben werden, a priori voraussetzen muss"], so kann ich mir diese Möglichkeit sehr wohl vorstellen". ${ }^{1}$ ) In dieser Darstellang fliessen somit zwei verschiedene Gesichtspunkte zasammen, erstens die Untersuchung der Erkenntnismittel, der Anschaungsformen and Verstandesbegriffe, sofern sie als Erkenntnismittel bei der Bildung der Erfahrang fungieren, zweitens die Voraussetzung, dass diese Erkenntnismittel nichts als "Beschaffenheit anseres Anschaungsvermögens" and Regeln des Verstandes in ans sind. Das heisst aber: Die erkenntniskritische Frage nach der Funktion der Erkenntnismittel fliesst bei Kant zwar nicht mit der alten Frage nach dem Sein, aber doch ohne weiteres mit der sorgsam davon za trennenden Frage nach der Herkanft dieser Erkenntnismittel zasammen.

Dies. lässt sich an dem Begriffe des a priori leicht deatlich machen. Die erkenntniskritische Bedentang des a priori liegt ganz einfach in den folgenden beiden Thatsachen beschlossen.

1) Krit. d. r. V. ed. Kehrbach, S. 18. 
Erstens: Ich kann keine Vorstellnngen haben ausser in zeitlicher Folge; ich kann keine Gegenstände vorstellen ausser im Raum; ich kann keine Veränderangen dieser Gegenstände vorstellen, ansser mittelst des Begriffs der Ursache a.s. w. Raum, Zeit, Kausalität etc. liegen also all meinem besonderen Erkennen za Grunde, sie sind notwendige Grandlagen aller Erfahrung. Das heisst: Während sich z. B. der Begriff eines Valkans, einer Sonnenfinsternis, einer Tiergattung erst nach Aufbau des Weltbildes und nach Erkenntnis vieler Einzelheiten der Erfahrang herausstellt, kann die Erfahrung selbst, der Aufbau des Weltbildes im einzelnen wie im ganzen gar nicht ohne Anwendang der apriorischen Formen zu stande kommen.

Zweitens: Von allen nach der Erfahrang gebildeten, bezw. ans der Erfahr ung geschöpften Begriffen kann ich niemals bindende Schlusse anf weitere Erfahrung ziehen. Ich kann darans, dass heate Vulkane speien, niemals folgern, dass sie immer speien werden und kann daraus, dass mir heute ein bestimmter Knochenban für eine Tierart charakteristisch erscheint, niemals mit Bestimmtheit schliessen, es könne nicht etwa eine andere, mir nnbekannte Tierart denselben Knochenbau besessen haben. Ganz anders ist dies bei Schlussen, die auf Grund der apriorischen Formen gezogen werden. Da kann ich, wenn ich einmal bewiesen habe, dass $\frac{a b}{2}$ den Inhalt des Dreiecks ausmacht, nicht im mindesten zweifeln, dass dieser Satz fur jedes mir vorkommende Dreieck gilt; und wenn ich $r^{2} \pi$ als Formel des Kreises bestimmt habe, so bin ich ausser Zweifel, dass diese Formel für keine andere Figur gilt. Dieser Geltangswert charakterisiert das a priori und macht seine wissenschaftliche Bedentang aus.

Diesem Geltangswert aber steht der Empirist ratlos gegentuber. „Waram ist in manchen Fällen ein einziges Beispiel zu einer vollständigen Indaktion hinreichend, während in anderen Fällen Myriaden ubereinstimmender Fälle ohne eine einzige bekannte oder nur vermatete Ausnahme einen so kleinen Schritt zar Festsetzang eines allgemeinen Urteils than?" So fragt J. St. Mill; and er hat keine Ahnung davon, dass die Antwort auf diese Frage mehr als ein halbes Jahrhundert vor ihm bereits gegeben worden ist. Er meint: „Wer diese Frage beantworten kann, rersteht mehr von der Philosophie der Logik, als der erste Weise des Altertums; er hätte das grosse Problem der Induktion gelöst.("1) Das muss ja wohl sein, dass

1) System der dedaktiven und induktiven Logik, tibers. $\nabla$. Schiel. 8. deutsche Aafl. I, S. 371. 
Kant von dieser Frage mehr verstanden hat, als der erste Weise des Altertums, denn jedenfalls hat er sie gelöst.

$\mathrm{Da}$ diese Seite der Kantischen Philosophie uber empirischen and psychologischen Einzelerwägangen noch immer von vielen verkannt wird, so sei die Art, wie sich unsere Erfahrang konstitaiert, an ein paar elementaren Beispielen illustriert. Wir haben z. B. die Wahrnehmung einer Orange. Worans besteht diese? Ans einer Summe von Gesichts- und ev. Tast- und Gerachsempfindungen, denen sich wohl Erimnerangen von Geschmacksempfindungen zagesellen mögen. Aber es ist nicht die einfache Summe dieser Empfindungen, sondern der an einer bestimmten Raumstelle vorgestellte Komplex derselben, der die Wahrnehmung ausmacht. Und diesen Komplex stellen wir, ebendamit, dass wir ihn wahrnehmen, als ein materielles Etwas, als eine Sabstanz vor. Substanz! Die sehen and fuhlen and schmecken wir nicht; was wir sehen and fuhlen and schmecken, sind sinnliche Empfindungen. Aber wir haben keine Wahl; wir m tussen diesen Empfindungen jenen Gedanken einer Substanz unterlegen, and erst damit, dass wir dies thun, nehmen wir die Orange als Ding wahr. Thäten wir es nicht, so wirde sie uns als ein Sinnentrag erscheinen. In dieser Thatsache, dass wir nur da, wo wir den Substanzgedanken zufügen, von Dingen reden können, oder, umgekehrt ausgedrückt, dass wir nur dann wirkliche Dinge wahrnehmen, wenn der Substanzgedanke untergelegt ist, liegt die Bedentang des Substanzgedankens als Erkenntnismittels and zwar als eines für all'e dingliche Wahrnehmung notwendigen Erkenntnismittels. Die besondere Frage, unter welchen besonderen Umständen wir den Substanzgedanken zuthan, unter welch anderen Umständen wir von Sinnentrug reden, berthrt obige Frage nicht; das ist eine Frage der Einzelforschung.

Die Entdeckang Kants, dass erst der zatretende Gedanke eine Wahrnehmung zur objektiven Wahrnehmung mache, ist jedoch nicht bloss "transcendental“ d. k. als notwendige Voraussetzung möglicher Erfahrung zu erweisen; man kann die Probe bei einiger Aufmerksamkeit selber machen, nicht bloss in Bezug auf den Substanzgedanken, sondern auch in anderen Fällen, bei wahren wie bei irrtumlichen Wahrnehmungen; ja man kann das Experiment willkurlich machen. Bekanntlich nimmt man oft wahr, dass der eigne Zug abfährt, während der neben uns stehende stille steht, und muss nach einem Blick anf die ubrige Umgebung den Sachverhalt umgekehrt denken. Aber sobald diese Korrektar in Gedanken da ist, ist anch 
meist die Wahrnehmung selber umgekehrt. Bei einiger Übang nan, freilich nicht immer gleich, gelingt es, dass man, ohne das Auge za wenden, durch blosse Umschaltang des Gedankens anch die effektive Wahrnehmung umschalten kann, zwei-, drei-, viermal während des langsamen Vorbeifahrens eines Zuges.

Ebenso kann man bekanntlich ein regelmässig geordnetes Tapetenmuster je nach Belieben aus krenzweisen Reihen, aus Dreiecken, aus Rhomben, ev. auch aus Quadraten bestehend nicht nur denken, sondern auch wahrnehmen. Mit einiger Mühe bringt man es an geeigneten Punkten sogar fertig, dass man nicht die Sonne hinter dem Bergrand nntergehen, sondern den Bergrand sich vor die Sonne heben sieht, also die der Kopernikanischen Thatsache entsprechende Wahrnehmung erhält; und dies geschieht offenbar vermöge blossen Hineinlegens des Gedankens. Wenn dies Verhalten des Geistes einerseits daza fuhrt, dass wir anf natürlichem wie anf geistigem Gebiete oft irrigerweise etwas unmittelbar wahrzunehmen glauben, was erst durch unsern Gedanken, den wir ohne Wissen hereingelegt baben, unr Wahrnehmung geworden ist, so belegt es doch anf der anderen Seite die Thatsache, dass unsere Vorstellungen erst durch die Denkzathat za gegenständlichen Wahrnehmungen werden.

Aber wenn wir dies festgestellt haben, so bleibt uns noch tibrig, eine Beobachtang za betonen, die Kant wohl gemacht, aber in ihrer Konsequenz nicht beachtet hat. Sie bezieht sich auf die Frage: Was drücken wir durch die Anwendung des Substanzgedankens als Erkenntnismittel aus? Darauf lautet die Antwort: Sobald wir den Substanzgedanken hinzuthun, wird uns der betreffende Vorstellnngskomplex unweigerlich zur Wahrnehmung eines thatsächlich ansser uns und unabhängig von der zufälligen Einzel-Wahrnehmung existierenden Dings. Die Wahrnebmung sagt mittelst des in ihr liegenden Substanzgedankens ans, dass das wahrgenommene Ding unabhängig von ihr da are. Denn Substanz heisst ja das Beharrliche in der Zeit, darauf verschiedene Einzelwahrnehmungen einheitlich als auf dasselbe Ding bezogen werden.

Durch den in der Wahrnehmung liegenden Substanzgedanken wird, ohne dass wir das merken, dem subjektiven und wieder verschwindenden Empfindungsgehalt der Gegenstand selbst als der Subjektivität entruckt untergelegt. Diese Art der Objektivität besteht allen Ausfluchten zum Trotz als notwendige Bedingung aller Erfahrung. Und hieraus allein ergiebt sich, wie bodenlos es ist, 
wenn sogar ein Helmholtz behaupten kann, ein. idealistisches Weltbild sei konsequent durchfuhrbar, wenn es auch eine praktisch sehr zweifelhafte Hypothese sei. Ein Weltbild ist vielmehr garnicht möglich ohne Wahrnehmung, and wenn in der Wahrnehmung notwendig der Gedanke eines ausserhalb der Wabrnehmung Beharrenden steckt, so kann dieses Beharrende nicht in die Subjektivität zaruckbezogen werden, ohne die Wahrnehmung selbst und damit jedes Weltbild aufzabeben.

Ganz dasselbe zeigt sich, wenn wir den objektiven Kausalgedanken betrachten.

Nehmen wir wahr, dass das schöne runde, gelbe Orangenbild verschwindet, und nur ein Hänfchen Schalen and Scheiben vor uns liegt! In diesem Fallé haben wir zunächst nichts als eine Reihenfolge subjektiver Empfindungen, bezw. einzelner verschiedener Wahrnehmungen. Aber damit begnügen wir uns nicht. Wir sagen vielmebr, die Orange ist geschält und zerteilt worden. Wir beziehen somit die Reihe verschiedener Empfindungen bezw. Wahrnehmungen auf einen einheitlichen, ausser ons stattfindenden Vorgang. Wir behalten trotz der verschiedenen Inhalte die identische Beziehang auf denselben Gegenstand bei and konstatieren: Der Gegenstand ist anders geworden. Üherall da aber, wo wir solche Veränderung an demselben Gegenstand konstatieren, denken wir, ebendamit dass wir dies thun, einen neuen Gedanken hinza, den nämlich, dass der Zeitreihe der geänderten Empfindungen e in e Änderung im Gegenstande selbst entspricht. Aber wie können wir behaupten, wir hätten denselben Gegenstand vor uns, wäbrend doch die Empfindungsinbalte ganz anders sind? Nar dadurch, dass wir hinzadenken, es sei eine gegenständlicbe Beziehung vorhanden, welche die Änderung an demselben Gegenstand bedingt. Diese gegenständliche Beziehung aber nennen wir Ursache. Nur vermöge des Hinzadenkens des Ursachgedankens ist es somit möglich, eine Veränderang von Wahrnebmungen als gegenständliche Veränderung von Dingen aufzufassen; und hieraus stammt die unausweichliche Nötigang, nunmehr nach den bestimmten Ursachen der bestimmten Veränderungen za fragen. Der Kausalgedanke kommt also nicht etwa erst zur Wahrnehmung eines Geschehens nachträglich hinzu; ${ }^{1}$ ) sondern die Wahrnehmung des Ge-

1) Hier gilt es den Ausdruck „Wahrnehmung der Veränderung“ beachten. Kant sagt (Kehrbach S. 181) der Begriff der Veränderang liege nicht in der Wahrnehmung verschiedener Zustände, sondern werde hinzugedaoht. 
schehens selbst als eines gegenständlichen Geschehens ist nur auf Grand des Kansalgedankens möglich. Der Kausalgedanke selbst ist in seinem Kern nichts als die Festhaltung der identischen Beziehang trotz der Verschiedenheit der Inhalte.

Daram müssen wir auch hier konstatieren: Damit, dass wir eine Reihe wechselnder Empfindungen als Wechsel in der Wahrnehmung desselben Dinges, also als Veränderung des Dinges selbst auffassen, sagen wir, dass diese Veränderung sich wirklich ansser uns und unabhängig von unserer zufälligen Wahrnehmung dieser Veränderang vollzogen habe. Und auch hier ist diese Feststellung von der anderen Frage za trennen, ob wir da vielleicht im einzelnen geirrt und vielleicht etwas als Veränderung eines Dinges angesehen haben, was vielleicht nur eine Veränderung an einem subjektiven Medium der Beobachtung war.

Diese und die dem Gesagten analogen thatsächlichen, für das Verständnis der Erfabrang grundlegenden und massgebenden Feststellangen mussen wir festhalten, denn sie fuhren allein ans dem Irrlichtelieren phantastischer Spekulation, die schon Kant selbst in der Kritik der Paralogismen and Gottesbeweise abgefertigt hat, anf den klaren und sicheren Boden wissenschaftlichen Denkens und Forschens. Sie geben die Lösung eines Problems, um das die Jahrtausende gerungen haben.

Dass aber diese Lösung keinen allgemeinen Anklang gefunden hat, ja noch hente von weiten Kreisen nicht gewurdigt wird, hat seinen Grand in dem oben erwähnten Umstande, dass Kant die Frage nach dem Geltungswert des objektiven Apriori nicht von der Frage nach seiner Herkunft za trennen vermag and letztere Frage voreilig and widerspruchsvoll entscheidet. Nachdem er z. B. in durchaus exakter Weise in der sog. "metaphysischen Erörterung" des Raums erwiesen hat, dass der Ranm nicht erst nachlräglich, d. h. als "empirischer Begriff" von äusseren Erfahrungen abgezogen sei, sondern diesen Erfahrangen za Grande liege, und nachdem er daraus in der "transcendentalen Erörterang" die Möglichkeit, apriorische Schliusse von allgemeiner Geltung za ziehen, also die Möglichkeit der Geometrie als Wissenschaft begriundet hat, springt er ohne weiteres za folgenden "Schlussen" uber: "Der Raum stellt keine Eigenschaften (oder Verhältnisse) irgend einiger Dinge an sich

Das ist keineswegs ein Widerspruch zu dem eben Gesagten. Denn die Wahrnehmung verschi $\operatorname{coden} \theta \mathrm{r}$ Zustände ist eben noch nicht die Wahrnehmung dieser Zustände als eines $\theta$ inzigen objektiven Vorgangs. 
vor," sondern ist "nichts anderes als die Form aller Erscheinungen äusserer Sinne, d. i. die subjektive Bedingung der Sinnlichkeit, anter der allein uns änssere Anschaunng möglich ist". Ganz ebenso verfährt er, in Bezng anf die Zeit, nachdem er sie ganz vortrefflich als eine allen, nicht bloss allen äusseren Anschaungen notwendige Grundlage dargelegt, und betont hat, dass die Wissenschaft der Bewegangslehre nur anf Grandlage der Zeitvorstellang apriorische Schlisse ziehen kann. Und dasselbe Spiel wiederholt sich betreffs der Kategorien. Der richtige Gedanke, dass die Kategorien Bedingungen a priori sind, die der Bildang der Erfahrung za Grande liegen, also Bedingangen möglicher Erfahrung sind, fuhrt sofort za dem voreiligen Schluss, dass sie in "der Natar anseres Gemütes" als "subjektive Grunde"1) der Einheit liegen.

Freilich, diese Schlussfolgerung liegt nahe genug. Wenn man einmal erkannt hat, dass die Erfahrung nicht fertig in uns bereinfliesst, sondern von uns geschaffen werden muss, so scheint es ohne weiteres, als mussten die Erkenntnismittel, durch die wir die Erfahrung schaffen, auch in uns ihren Ursprung nehmen, also vom "Gemilt" aus sich heraus hinzugegeben werden.

Und es ist leider Thatsache, dass gerade dieser Schluss in der Geschichte der Philosophie eine weit bedentsamere Rolle spielt, als Kants wertrollere methodische Untersuchung, hinter der er sich als Verlegenheitsanskunft aufbant. Sowohl bei den Fortbildnern Kants von Fichte bis Hegel als anch bei Gegnern des Mannes bildet gerade diese Schlussfolgerang den Angel, um den sich die Haupterörterang dreht.

Aber dieser Schluss ist erstens an sich nicht notwendig, and zweitens fuhrt er za Widerspruchen, die unlösbar bleiben, wenn man ihn festhält.

Der Erweis, dass der Schluss nicht notwiendig ist, ist am besten dadurch za erbringen, dass man eine andere Möglichkeit der Erklärung, bezw. der Herkanft obiger Begriffe zeigt. Um aber dazu zu gelangen, eine solche anderweitige Erklarang aufzusuchen, muss man die Widersprüche aufdecken, die sich infolge jener Annahme ergeben. Diese sind nun allerdings in der Hauptsache seit lange empfunden, aber meist in falscher Weise geltend gemacht worden. Man hat gesagt, dass eine Objektivität, die durch unser Denken geschaffen werde, doch keine Objektivität der Dinge selbst sei,

1) Kehrb. S. 134. 
dass also die "Erscheinung" im Grande Schein sei. Mit diesem Einwande aber macht man Kants fehlerhafte Schlussfolgerang selbst mit, supponiert also mit ihm, dass die Denkformen, durch die wir Erfahrung schaffen, auch im Subjekt entspringen mussen. Gegen solche Formalierung des Einwands hat darum der Kantianer leichtes. Spiel. Er braucht bloss anf die von Kant festgestellte objektive Gesetzmässigkeit hinzaweisen, die nur unter genannter Voraussetzung möglich ist, and die wahrlich kein Schein ist. Er kann sagen: Wir können doch nie hoffen and beanspruchen, dass die Dinge, wie sie an sich, d. h. unabhängig von unserer Erkenntnis sind, in uns hereinspazieren. Im Gegenteil, gerade dann, wenn dies wäre, so wären sie Schein und nicht ausser uns befindliche wirkliche Dinge. Dass aber die Erkenntnisbestimmungen derselben nar in uns and nirgends anders sein können, ist so selbstverständlich, dass darüber kein Wort za verlieren ist. Und hiergegen bringt obiger Einwand nar eine Behauptung, aber keinen Beweis. - Wenn also Plechanow meint, Kant damit zu widerlegen, dass er ihm sagt: "The proof of the pudding is the eating!" so trifft er ihn an ganz falscher Stelle. Das lengnet ja Kant in keiner Weise; gerade er betont ja, dass Empfindung „die wirkliche Gegenwart des Dinges" anzeige. Und gerade er hat ja die phantastischen Versuche, mit Umgehung der Bedingangen der Sinnlichkeit za "Dingen an sich" za gelangen, mit aller Energie bekämpft and hat die Wirklichkeit der Dinge in der ersten ebenso wie in der zweiten Aaflage festgebalten. ${ }^{1}$ )

Der Widerspruch, den sich Kant zu schulden kommen lässt, besteht nicht darin, dass er einerseits subjektive Vorstellangen, andererseits Dinge annähme, die davon unabbängig sind. Der Widersprach besteht nur darin, dass er den Ursprung nicht nur derjenigen Vorstellangen, welche wie Möglichkeit, Notwendigkeit, Bejahang; Verneinung etc. bei der Bildang der Dingrorstellangen notwendig sind, aus der "Subjektivität des Gemüts" als ihrem "Quell" herleitet, sondern dass er mit diesen Vorstellungen eine ganz andere Reihe von

1) Wenn Plechanow den alten Gedanken wieder aufwärmt, in der 1. Auft. stelle sich Kant zu den „Dingen an sich" anders als in der zweiten, so verwechselt er eine blosse Frontveränderung der Polemik mit einer saohlichen Veränderung. In der 1. Aufl. streitet Kant bloss wider diejenigen, welche aus blosser Vernunft an die Dinge gelangen wollen, in der 2. Auf. auch wider die, welche behauptet haben, er sei materialer Idealist. Die Stelle (Kehrb. 283) aus der 1. Autl. (S. 251), dass ans dem Begriffe einer Erscheinung tolge, dass ihr etwas entspreche, was nicht Erscheinung sei, zeigt aufs deutlichste, dass er auch hier durchaus keine material-idealistische Position eingenommen hat. 
Vorstellungen unterschiedslos zusammenwirft, nämlich diejenigen, welche als Bestimmungen der Naturerfahrung verwendet werden. Wenn wir sagen, ein Ding ist „möglicherweise“ vorhanden, so geben wir damit kein Prädikat eines Dinges, sondern nar ein Verhalten unseres Erkenntnisvermögens an. Wenn wir aber urteilen: „Das Ding beharrt in der Zeit", oder "das Ding verändert sich", so geben wir Aussagen uber die Dinge selbst; und wenn diese Aussagen ebenfalls „in der Subjektivität unseres Genuts" ihren Ursprung hätten, so wären sie nicht möglich. Sobald wir sagen mussen, ein Flecken, den wir am Objekte sehen, gehöre, dem Objekte selbst an, können wir nicht sagen. er stamme aus der, Natar der Brille, durch die wir sehen. Und ebenso umgekehrt. Hier, in der Erkenntnisbeziehung, nicht in dem Gegensatz Ding-Vorstellung liegt der Widerspruch. Das verkennt Plechanow, wenn er seinen Einwand z. B. so formuliert: „Bei Kant haben die Gesetze a priori keinen objektiven Wert, oder mit anderen Worten, sie. besitzen nar Geltang für die Phänomene nicht für die Dinge an sich."1) Der Kantianer muss denken: Ist denn der Manu nicht klug, „der einem Kant gerade das vor der Nase wegleugnet, was dieser zuerst klar bewiesen hat, den objektiven Geltangswert der Gesetze a priori?" -- Und was soll erkenntniskritisch bedenten „Dinge an sich?": Das wären Dinge, die erfalst werden sollen, abgesehn von Bedingungen unseres Erkennens; and gerade dieses spekulative Suchen nach dem Stein der Weisen haben ja Kants Forschangen unmöglich gemacht. - Also, Plechanows Einwand ist fur einen Menschen, der Kant versteht, nirgends za fassen. Hätte er gemerkt, dass Kant selbst jenen metaphysischen Gegensatz im Ange hat zwischen einem Dinge, das wir naturlich erkennen, and einem Dinge, das wir hinter unseren Erkenntnisbedingangen heram (durch blossen Verstand) erkennen wo!len, so hätte er seinen Einwand anders formuliert. Er müsste nach dem Gesagten lanten: „Kant zeigt, aus welchen Komponenten das objektiv gultige, anf Naturdinge angewandte Urteil geistig zustande kommt, aber er ubersieht, dass Aussagen, die von dem Dinge selbst gelten sollen, unmöglich ihren Quell rein in der Snbjektivität des Gemüts haben können." Gewiss will Plechanow in der Sache nichts anderes als dies behaupten; wenn er vom "Ding an sich" spricht, meint er eben das Natording; aber seine Verkennung der positiven Leistang Kants veranlasst ihn za Folmolierangen, die gerade diese Leistang in Frage stellen and er macht dadurch seine Einwände wirkungslos.

1) N. Z. $1898 / 99$ No. 20 , S. 629. 
Sind wir uns aber darkber klar geworden, dass Kant in seiner Kategorientafel von vorn herein nicht zwischen solchen Verstandesformen scheidet, welche bloss anser Verhalten bei Bildung der Erfahrung angehen, und solchen, welche zu Prädikaten der Natur selber werden, sondern beide unterschiedlos durcheinanderwirft, dann stellt uns diese Unterscheidung vor ein nenes Problem. Wir fragen nicht mehr metaphysisch, wie wir von Dingen an sich za Erscheinungen kommen oder umgekehrt, sondern wir fragen erkenntniskritisch nach dem Unterschiede von zwei ganz verschiedenen Arten objektiver Gultigkeit.

Diesen Unterschied wollen wir uns noch an einigen Beispielen anschanlich machen. Wenn wir die Höhe and Breite eines Steines messen, und daraus den Kubikinhalt berechnen oder dergleichen, so sind die verschiedenen Verfabren, die wir dabei anwenden, objektiv guiltig, aber eben nur objektiv notwendig für das Verfahren selbst. Dagegen nur das Resultat ist gültig für den Naturgegenstand: den Stein. Addition, Maltiplikation, Potenzierang, Wurzelauszieben etc. sind noiwendige und objektiv gültige Operationen für das Verfahren, aber sie sind in keiner Weise objektiv gültig für Naturgegenstände. Was soll $\sqrt{ }-1$, anf Natur bezogen, bedeuten? Nichts, reinen Unsinn! Daraus ergiebt sich, dass wir bei unseren notwendigen und objektiven Begriffen nicht bloss den Geltungswert festzustellen haben, und nicht etwa bloss, wie Kant that, zwischen Sinnlichkeit and Verstand za scheiden haben, sondern dass wir weiterhin prüfen mussen, für welche Art von Objektivität die betr. Begriffe gultig sind. Es muss also neben der Beziehnngsart anch der Beziebungsort eines Begriffs erkenntniskritisch untersucht werden. Wenn sich also herausgestellt hat, dass Möglichkeit, Notwendigkeit, Bejahung, Verneinung etc. nur unser Verfahren in Bezug auf Gegenstände, Raum, Zeit, Kausalität, Substanzialität aber die äussere Nator selbst zu Beziehangsorten haben, so muss die Befugnis, von solchen Beziehungsarten zu prädizieren, besonders untersucht werden.

Daza reicht aber Rants Untersuchung nicht aus. Er begrundet die objektive Geltung ans der sog. transcenàentalen Einheit der Apperzeption, d. i. daraus, dass alle Begriffe in notwendigem Zusammenhang zam "Ich denke" in demselben Subjekt stehen mussen. Darin hat er insoweit vollkommen recht, als er zeigt, dass nar der notwendige, einheitliche Zusammenhang aller Erkenntnisse uns Wahrheit verburgt, dass wir also Irrtumer stets nur daraus erkennen können, dass irgendwelche Denkbestimmangen sich widersprechen. 
Aber genannten Unterschied kann er damit nicht begreiflich machen. Es bleibt schon ein Rätsel, wie neben der notwendigen Beziehnng anf das „Ich denke" noch eine „empirische Apperzeption" mit ihren vielfachen Irrtumern möglich ist. Es bleibt aber vor allem der Grand unbegreiflich, waram einige der apriorischen Denkbestimmangen konstitutive, andere, wenn wir so sagen durfen, bloss konstraktive Erkenntnismittel sind.

Das hieraus entspringende Problem lässt sich durch kein Dekret uber den Ursprang lösen, oder aus der Welt schaffen. Das Dekret, sie seien im Sabjekt entsprangen, schafft einen fast noch grösseren Widersprach, als das Dekret, sie stammten aus der Erfahrang. Letzteres enthält für den, der Kant durchdacht hat, freilich einen unansgleichbaren Widersprach. Denn wie können diejenigen Elemente erst Erzengnis der Erfahrang sein, darch welche Erfahrang ihrerseits erzengt werden muss. Ersteres aber führt za ganz baltlosen Versuchen, der Subjektivität durch ungeheuerliche Annahmen za entgehen. Dahin gehören das Dekret, dass der Quell dieser Formen nicht im Subjekt, sondern in einem generellen Ich, wie Fichte annimmt, oder in einem "Bewasstsein uberhaupt", wie Laas es nennt, za finden sei. ${ }^{1}$ ) Doch diese Ausflucht frachtet nichts. In der Erfahrang erscheint das Subjekt als Teil in der Kette der Erscheinungen, and zwar als recht kleiner Teil. Als das die Erfahrang gestaltende Subjekt hat es aber die ganze Natur in sich. Hat es nun die Elemente zar Gestaltung der Erfahrung aús sich genommen, als seine Erzengnisse, so mulsste seine Subjektivität einerseits die gesamte Objektivität thatsächlich umfassen, andererseits nar ein Teil darin sein. Das Bewasstsein uberhaupt oder das allgemeine Ich löst diesen Widersprach nar darch Flucht aus der Dämmerung ins Dankel, und lässt das Problem im Nebel eines mysteriösen Wortes verschwinden.

Anch das Verfahren Cohens und seiner engeren Anhänger genuggt hier nicht. So sehr wir diese Forscher gerade darin schätzen müssen, dass sie das eigentlich wissenschaftliche Fundament des Kantianismus, die Untersuchung der Erkenntnismittel ausgegraben and der zerfahrenen Philosophie der Gegenwart gegenuber mit zäher Energie endlich mehr und mehr zur Geltung gebracht haben, so wenig können wir es billigen, dass sie das in Frage kommende

1) Kants Analogien der Erfahrung S. 94 ff. Als ich diesen Passus s. Z. zu lesen begann, hielt ich ihn für bittere Ironie; aber leider zeigte sich, dass Laas blutigen Ernst machte. 
Problem geradezu ausschliessen wollen. Wenn freilich Cohen²) das Ding an sich für den Inbegriff aller wissenschaftlichen Erkenntnisse, bezw. als "Aufgabe" bezeichnet, so haben wir an sich gar nichts gegen eine solche Definition einzuwenden. Aber - sie bezeichnet weder Kants Ding an sich, noch löst sie das darch ihn offen gelassene Problem. Sie ist eine Bestimmang, die nach Lösung des Problems ganz vortrefflich ist, vor dessen Lösung angewandt aber einer spanischen Wand gleicht, die es verdecken soll. Und dabei ist dieser Verdeckangsversuch umsonst. Anch bei Cohen erscheint das Bewusstsein als "Quell der Erfahrung", aber um dem Schein des Subjektiven zu entwischen, meint er den „Inbegriff der Mittel und Methoden," welche wissenschaftliche Erkenntnis ausmachen. ${ }^{2}$ ) Er geht also um den Fragepunkt heram, and behält die Subjektivität im Grande bei, ohne es Wort haben za wollen. Ebenso sagt Natorp in seinem neuesten Buch: ${ }^{3}$ ) Die Bestimmungen, in denen man das Gegebene zu fassen versucht, "stellen sich bei näherer Betrachtang als Denkbestimmungen heraus, die als solche nichts Gegebenes, sondern eigne Gestaltungen des Denkens sind." Auch er sieht also da kein Problem, sondern lässt ganz rahig die notwendigen Bedingungen objektiver Erfahrang ohne Unterschied eigne Gestaltangen des Denkens sein.

Alle diese Aufstellungen verkennen oder amgehen somit das Problem, um das es sich handelt. Das Problem haben wir erst gelöst, wenn wir zeigen können, dass wir Bewusstseinsvorgänge haben, die wir als wirkliche Relationen zwischen uns and den Dingen ansehen mussen, und dass die konstitutiv apriorischen Formen ohne Schädigung ihres Geltungswerts von ihnen abgeleitet gedacht werden können. Dann können wir begreiflicherweise dadurch, dass wir Bestimmungen, die ans diesen Relationen abgeleitet sind, auf die Dinge untereinander übertragen, gültige Urteile übèr diese Dinge selber fällen. Das ist dann genan so verständlich, wie es verständlich ist, dass wir die Höhe eines uns nnzugänglichen Turmes aus den Winkeln, die er mit einer uns zugänglichen Wegstrecke bildet, berechnen können.

Um eine derartige Lösung des Problems vorzubereiten, müssen wir zunächst einige besondere Funktionen anseres Bewasstseins ins

1) Kants Theorie der Erfahrung 2. A. S. 519.

2) Cohen a. a. O. S. 141 f.

3) Sozialpädagogik S. 25. 
Ange fassen, die Kant bereits betont hat, die Analyse and die Synthese. Wenn wir die Erfahrung, wie sie uns jeweils vorliegt, betrachten, so finden wir, dass jeder Fortschritt in ihr geschaffen wird mittelst Analyse irgend einer besonderen Einzelheit ans einem bisher noch ununterschiedenen Boden und durch Beziehnng dieser Einzelheit auf einen Zusammenhang, in den sie sich einheitlich einftgen lässt. So erscheint eine Landschaft zunächst in ganz allgemeinen Zugen vor unserem Ange. Erst aufmerksame Beobachtang lässt besondere Einzelheiten herportreten, and diese fixieren wir dann oft nicht ohne Mthe and Irrangen neben- and hintereinander, so dass ans endlich statt des Bewasstseins der verworrenen Fläche ein mehr oder minder gegliederter und geordneter Znsammenhang vor Augen steht.

So analysiert das $\mathrm{Ohr}$ ans dem Tongewire eines Orchesters die Töne eines Instrumentes and bringt diese als geordnete Melodie zom Bewusstsein. So analysieren wir, mit der Fingerspitze hin- und hergleitend, die Formen einer Fläche und bringen uns dieselben als Zusammenhang des Gegenstands zum Bewusstsein. Wir vollziehen also, indem wir analysieren, schon in der sinnlichen Beobachtung gewissermassen Abstraktionen, indem wir, ron allen anderen Bestandteilen des Untergrands absehend, bloss bestimmte Einzelheiten ins Ange fassen, und diese bringen wir dann synthetisch untereinander in Beziehnng.

Dabei ist uns folglich jedesmal nur diejenige Einzelheit, bezw. die Reihe der Einzelheiten deatlich bewusst, die wir gerade analysieren; diese Reihe wissen wir, aber wir beobachten dabei oft nicht einmal, dass wir das than, geschweige denn, wie wir das than So ergiebt sich uns bekanntlich das objektive Nebeneinanderbestehen bestimmter Dinge daraus, dass wir mit dem Ange oder dem Tastorgan nach Belieben wechselweise zwischen dem einen and dem anderen hin- and hergehen. Die Synthese „nebeneinander" vollzieht sich auf Grund der wechselseitigen Analyse. Wo wir diese nicht wechselseitig vollziehen können, wo vielmehr die Wahrnehmungen bloss zeitlich folgen, da reden wir nicht von nebeneinander bestehenden Dingen, sondern von Vorgängen, die "nacheinander" folgen. Das than wir jederzeit unmittelbar and meist blitzartig schnell; aber das Bewasstsein, dass wir das so thun, steigt vielen Menschen ihr Lebenlang nicht auf. Dies Thun könnten wir, um es vom eigentlich unbewassten za unterscheiden, „unterbewasst" nennen. Bewusst wird es erst dann, wenn wir gelernt haben, die bei Analysiernng and Verkntipfang der objektiven Begriffe ausgettbte eigne Thätigkeit 
za untersuchen und ihrerseits begrifflich za machen ${ }^{1}$ ) das heisst, wenn wir nicht mehr Naturphilosophie and Metaphysik des Seins treiben, sondern Erkenntniskritik tben.

Nach diesen Vorbemerkungen kommen wir za dem Problem zaridck, das wir aufgestellt haben, und fragen, ob wir unter unseren Erkenntnisfunktionen solche haben, die von vornherein Beziehungen von Dingen za ans enthalten, oder genauer, ob Beziehungen in uns vorhanden sind, die wir notwendigerweise als Beziehungen von etwas Fremdem za uns deaten mussen, und ob wir hiervon Bestimmangen abzuleiten rermögen, die wir nachher als auf Beziehungen der Dinge entereinander übertragen auffassen müssen. Können wir das leisten, dann ist der Schluss gegeben, dass letztere Beziehungen auf dem Wege der ursprunglichen unterbewussten analytischen und synthetischen Thätigkeit abstrahiert und verknupft worden sind.

Solche Beziehangen aber, wie wir sie suchen, kennen wir seit lange - in unseren Emptindungsvorgängen. Diese gelten uns stets als unmittelbare Affektionen, als passive Veränderungen unseres Zustands als Beziehnng von etwas Fremdem za uns. Freilich, das muss betont werden, nur im lebendigen Empfindungsvorgang als einem Geschehen, kann solche Beziehung liegen, nicht bloss in dem nach Qualität und Intensität bestimmten Inhalte dieses Vorgangs. Der gesamte Empfindungsvorgang enthält weit mehr als diese, bereits durch Analyse aus ihm gewonnenen Elemente. Daranf weist schon Kant selbst. "Sinnliche Anschaunng," so sagt er, „enthält die Art, wie wir von Gegenständen affiziert werden." Damit sagt er, dass das Grundelement der sinnlichen Anschaunng, die Empfindung, die ja „die wirkliche Gegenwart des Gegenstandes voraussetzt", ${ }^{\text {,) }}$ als passives Affiziertwerden zam Bewusstsein kommt. „Affiziert werden" heisst aber, Einwirkungen versptiren, zeitliche Veränderung des eigenen Zustandes spüren, führt also den Kansalgedanken mit sich.

Es kann sich da höchstens um das Bedenken handeln, ob dieser Kausalgedanke erst nachträglich zam Empfinden hinzagedacht wird, oder ob er ursprünglich in ihm selber liegt and erst durch Analyse davon abgesondert wird.

$\mathrm{Da}$ wir nicht bei unserem ursprünglichen Bewasstsein, d. h. bei anserer ersten Empfindung Nachfrage halten können, so ist diese Frage empirisch nicht zu entscheiden. Die Analyse unseres heutigen Empfindens kann uns da keine volle Gewissheit geben. Denn es

1) „Die Synthesis anf Begriffe bringen“, Kehrbach, S. 95.

2) Kehrbach, S. 76 . 
bliebe, sobald wir sagen wollten, der Kansalgedanke sei ans der Empfindung abgeleitet, dennoch der Verảacht bestehn, dies könne eine Tänschang sein, indem diese nenerdings rorgenommene Analyse auf einer fur uns nicht mebr kontrolierbaren ursprunglichen Synthese ruhe.

Um dieses Dilemma za lösen, kommt uns aber Kants eigne Methode vortrefflich zn statten. Die ,transcendentale" Frage, die er stets als methodischen Entscheidangsgrand stellt, lantet: Ergiebt sich eine gemachte Voraussetzung als Bedingung möglicher Erfahrung? Ist das der Fall, so lautet die Folgerang: Was sich als Bedingang möglicher Erfahrung herausstellt, wird ebendamit zar notwendigen Bedingang wirklicher Erfahrung.

Non haben wir bereits gesehen, dass gewisse apriorische Formen notwendige Bestandteile der Erfahrang sind; und wir haben dargethan, dass mit diesem thatsächlichen Geltangswert die Annahme nicht stimmt, dass sie aus der reinen Subjektivität des "Gemults" stammen. Stammen sie aber nicht ans dem „Gemute", and mussen sie dennoch apriorisch sein, so bleibt nar die Wahl, dass sie von einem arsprünglichen Erfahrangselemente abgeleitet sind. Da wir nun kein anderes Erfahrungselement haben, von dem sie abgeleitet zu denken wären, als den Empfindangsvorgang, so ergiebt sich, dass die konstitativ-apriorischen Formen ursprünglich in ihr liegen and erst aus ihr abstrahiert sein mussen.

Es wäre denn doch auch allzu seltsam, dass wir nor in späterer Erfabrung, falls wir einen ungewohnten Ton hören, einen anerwarteten Lichteindrack haben, eine plötzliche Tastempfindung rerspüren, sofort fragen sollten, woher das kommt, wenn nicht der Empfindangsvorgang selbst durch eine in ihm liegende Eigenart diese Frage an die Hand gäbe. - Und ferner: Falls das Kansalbewusstsein im Empfinden liegt and von ihm abstrahiert ist, so ist es sebr begreiflich, dass wir uberall da, wo wir Veränderangen wahrnehmen, a priori eine Ursache postalieren müssen, während amgekehrt, wenn die Empfindung nicht selber das Kansalbewusstsein enthält, gar kein Grund abzusehen ist, warum wir sie durch Zadenken des Kausalgedankens zum Bewusstsein eines Affiziertwerdens, d. h. za einem Vorgange, der eine Ursache fordert, machen mulssten, oder auch nur machen könnten,

Wir mulssen daher za dem Schlusse kommen, dașs die konstitativ-apriorischen Formen aus dem Empfindungsrorgang abgeleitet 
werden müssen, dass also die apriorisch-synthetischen Urteile uber Dinge - bezw. die Bildung der Erfabrung - auf einer Übertragung ursprunglich unterbewusster Apstraktionen auf das Verhältnis zwischen Dingen beruhen.

Die Frage, wie dies geschehen möge, ist nun von besonderer, im einzelnen nicht mehr "transcendentaler" d. b. erkenntniskritischer, sondern psychologischer, bezw. psychologisch-physiologischer Art. Aber dennoch ist es gut, wenigstens einige allgemeine Gesichtspunkte zu geben. Denn die Möglichkeit, sich die Erfahrung in dieser Weise aufgebaut zu denken, bildet gleichsam die praktische Probe auf obige Schlussfolgerang.

Ist die Empfindung schon ursprünglich Bewusstsein eines Affiziertwerdens, also Relation eines Fremden zu uns, so muss die Reaktion des Bewasstseins darin bestehen, dass dies Fremde bestimmt werde. Allein za seiner Bestimmung haben wir zunächst - wenn wir davon absehen, dass Empfindungsvorgänge wohl schon ursprünglich eine unterbewusste Grösse haben mögen - nur Qualität und Intensität der Empfindung. Also kann das Fremde zunächst als bloss durch diesen Inhalt bestimmt gedacht werden. Es ist dem Bewusstsein nichts als das in dieser bestimmten Weise Affizierende: so können wir den Thatbestand der ursprünglichen Synthese bezeichnen.

Nan kommen weitere Affektionen. Nehmen wir zunächst der Einfachbeit balber solche von gleicher Qualität und Intensität. Sie werden notwendig auf dasselbe Fremde, also identisch bezogen. Und diese identische Beziehnng besagt: es ist dieselbe gegenständlich beharrende Ursache, die auf uns verschiedenemale die gleiche Wirkung ansübt. Dieses und nichts anderes ist der Substanzgedanke. Dieser Gedanke ist somit nicht etwa rom Kausalgedanken verschieden; die dauernde oder gleichartig wiederkehrende Wirkung auf uns stellt uns vielmehr durch ihn eine gleichartige und dauernde Ursache dieser Wirkang vor das Bewasstsein.

Muss das Bewusstsein ursprunglich die gleichartig wiederkehrende Empfindang notwendig auf dasselbe beharrende Etwas, d. h. dieselbe Substanz beziehen, so muss es andererseits eine andersartige Empfindung ursprunglich auf eine andere Substanz beziehen. Und wenn nun die Beobachtung von dem einen zam andern Dinge unterschiedslos hin- und hergehen kann, dann wird, wie erwähnt, unmittelbar die ursprüngliche Beziehung eines Fremden, eines "ausser uns" auf das Verhältnis der beiden Substanzen übertragen. Sie werden als aussereinander bestimmt. Damit aber treten sie in gegen- 
seitige Beziehung in einer ausser uns vorgestellten Einheit - dem Ra a m.

Damit befinden wir uns sofort anf gewohntem Erfahrangsboden.

Sobald viele derartige Beziehungen auf verschiedene aussereinander befindliche Substanzen fixiert sind, gewinnt der Raum seine Ausfullang and seine Bestimmtheit. Oben and anten, neben, vorn and hinten, Grösse and Mass treten ins Bewasstsein. Und nun kann die Auswertang immer weiter gehen, indem jeder neue Inhalt, der neben oder hinter den bereits fixierten Stellen bezogen wird, immer wieder in dieselbe Relation des Aussenseins za den bereits fixierten Substanzen tritt. Endlich kann bei entwickeltem Bewusstsein diese Fixation neaer Raumstellen obne Empfindungsgrandlage bloss in Gedanken weiter gehen and fuhrt so zur Vorstellang von der Endlosigkeit des Raums.

Ist dann, beilänfig bemerkt, der Raum einigermassen derart bestimmt and ansgefullt, so können die neuen Empfindungen nicht mehr allesamt an verschiedenen Raumstellen fixiert werden. Ursprilnglich ist es denkbar, dass z. B. eine Gesichtsempfindang an einer Substanz, eine Tastempfindung an einer anderen Sabstanz fixiert wird. Mit der zanehmenden Ausfullung des Raums geht das nicht mehr. Die Empfindungen verschiedener Sinne müssen an denselben Raumstellen verbunden fixiert werden; die Vorstellang von Substanzen mit mehreren Eigenschaften, d. h. von Ursachskomplexen, die mehrere Empfindungsarten bewirkt haben, tritt ein. In steter Korrektur and Umordnung wird so das Weltbild geschaffen.

Eine andere Art der Beziehang schiebt sich von Anfang an herein, wo Empfindungsreihen in der Weise hervortreten, dass wir nicht von der einen zar anderen abwechselnd ubergehen können. Jetzt besteht, wie wir gesehen haben, die Notwendigkeit, objektive Vorgänge gegenständliche Veränderangen $z a$ stataieren. ${ }^{1}$ ) Diese Notwendigkeit aber setzt voraus, dass das unmittelbare und subjektive Bewusstsein der Folge von Empfindungen, vermöge dessen allein schon die Znsammenfassung fruherer and jetziger Empfindungen in dem Gedanken der Sabstanz möglich war, als objektive Folge von Vorgängen, also als objektiv zeitliches Geschehen aufgefasst wird. Die Zeit also, ursprünglich unterbewusste Form der ursprünglichen Relation der Empfindungsvorgänge, wird jetzt gegenständlicb. Und damit ist, wie

1) Dass sehr wahrscheinlich zuerst das Bewegte, nioht das Ruhende die Aufmerksamkeit erregt, ficht obige Darstellıng nicht an. Diese will nur schematisch die Grundbeziehungen in ihrer Möglichkeit erörtern. 
wir gesehen haben, eine Identität des substanziellen Zusammenhangs trotz gleichzeitiger Nichtidentität bestimmt, ein Widersprach, der seine Lösung in der Nachfrage nach der Ursache der Ändernng findet. Aber diese Ursache kann nicht in dem Vorgang selber liegen, sondern geradeso, wie wir die eigne Affektion im Empfinden auf etwas ausser uns beziehen mussen, so mussen wir die Affektion des ausser uns bestimmten Dings in einem erst ausser demselben bestimmten oder za bestimmenden suchen. Wir ubertragen also wiederam eine Besonderheit der arsprünglichen Relation des Empfindungsvorgangs auf die Relation zwischen äusseren Dingen bezw. Vorgängen.

Und hierdurch entsteht der Gedanke des Kausalzusammen. hangs, der später, zu wissenschaftlicher Klarheit erhoben, die Aufgabe stellt, dass jede Veränderang, welcher Art sie auch sei, nach Mass, Richtang und Eigenart zareichend zu bestimmen sei.

Wie sich nun diese Analysen und Synthesen im einzelnen vollziehen, das za erörtern, müssen wir uns versagen. Die physiologische Psychologie zeigt, dass der Erwerb schon des durftigsten Weltbildes ein sehr komplizierter Vorgang sein muss, dass tausendfache irrige Beziehangen und irrige Übertragangen vorkommen. Das Kind greift nach dem Mond, hält die verschiedensten Dinge für identisch etc., and wir Erwachsenen irren uns noch oft, wenn es gilt, auszumachen, woher ein Ton kam, ob ein Berg hinter oder neben dem andern ist a. dgl. Die Korrektur erfolgt da stets erst dann, wenn irgendwelche Widerspriiche zum Bewasstsein kommen. Das Auftauchen des Widersprachs ist in dieser Hinsicht eine vorwärtstreibende Instanz.

Die gegebenen Andeutungen durften für den vorliegenden Zweck gentugen. Es lag uns ja nur ob, die Möglichkeit zu zeigen, dass die apriorischen Fanktionen, wenn wir sie als analytische Abstraktionen aus dem Empfindungsvorgange als der urspringlichen Relation zwischen uns and der Aussenwelt anffassen, zu synthetisch apriorischer Verwendung für den Aufbau des Erfahrungsganzen genau ebenso geeignet sind, als wenn wir sie als Bestimmungen der Subjektivität ansehen.

Das sind sie aber in jeder Weise. Der Ranm liegt bei unserer Auffassung allem äusseren, die Zeit allem inneren und äusseren Sein and Geschehen ebenso zu Grande, wie bei Kant. Darum sind auch die Schlusse, die sich ans diesen Bedingungen der Erfabrang a priori ziehen lassen, ebenso bindend fur alle Erfahrang, wie sie es nach Kants Annahme sind. Die apriorischen Folgerangen der reinen Geometrie, der reinen Mathematik, der reinen Naturwissenschaft sind, 
wenn sie auf den genannten Bedingungen, und nicht, wie die Metamathematik auf einer Auswabl dieser Bedingungen, z. B. als Geometrie ohne Parallelensatz, aufgebaut sind,1) ebenso anwendbar auf Erfabrung, wie sie es bei Kants Voranssetzungen nur sein können.

In anderer Hinsicht dagegen ist unsere Ableitung, wie wir glauben, beträchtlich verständlicher, und mehr zur Erklärang geeignet, als diejenige Kants.

Sie lässt uns den Zusammenhang ron Natar and Leben weit unmittelbarer and einheitlicher begreifen; and vor allem beseitigt sie die ganze Reihe ron Rätseln und Widersprüchen, die bei Kants Annahme unvermeidlich sind und die, wie sich gezeigt hat, reranlasst haben, dass man an der bedeutsamen wissenschaftlichen Leistang Kants allzu oft achtlos vorïbergegangen ist.

Zunächst beseitigt sie die bei Kants Annahme unerklärbare Schwierigkeit, dass einige der aprioriscb notwendigen Denkfunktionen bloss konstruktiver Natur sind, d. h. bloss als Gerüste und Hilfswerkzeuge beim Aufban der Erfabrung gelten, wäbrend andere Denkfunktionen sich als konstitutiv, d. h. als Bausteine der Gegenstände selber herausstellen. Und damit wird auch das Rätsel gelöst, dass neben der transcendentalen Apperzeption, welche die objektiv gültige Einheit enthält, noch eine empirische Apperzeption rorbanden sein kann, in der Irrtümer vorzukommen vermögen.

Damit ist aber die Kluft zwischen Ding an sich und Erscheinung, die Cohen durch Umgehung nur scheinbar beseitigt hat, wirklich beseitigt. Der Materialismus, der die Beziehang der Vorstellungen auf wirkliche Dinge einfach postaliert, obne anderen Beweis als den cummon sense, der Empirismus eines Locke, der die primären Eigenschaften der Dinge „wahrnimmt", der positivistische Phänomenalismus, der alles mit der denkenden Verarbeitung von gegebenen Empfindungsinhalten abmacht, und zu keinen wirklichen Dingen kommt, der materiale Idealismus, der die Welt ans dem absoluten Geist hervorzaubert, der materiale Rationalismus, der den Knoten durchhaut and, wie Volkelt, den Verstand die Transsubjektivität gewährleisten lässt, endlich der Skeptizismus, der,

1) Systeme, die nicht die Gesamtheit der Erfahrungsbedingungen zu Grunde legen, mögen in sich selber äusserst konsequent sein; abor sie haben nichts mit Erfahrung zu thun. Sie sind eine mathematische Metaphysik, ganz analog der ehemaligen Vernunftmetaphysik, die ja auch, wenn konsequont durchgefïhrt, in sish einieitliche Systeme, oft wahre logische Kunstwerke ergiebt, die nur leider, auf Naturerfahrung bezogen, versagen. 
wie bei Helmholtz ${ }^{1}$ ), die idealistischen Konzeptionen für unwiderlegbar, wenn auch für praktisch ungeeignet hält: sie alle sind, wenn anders unsere Ableitung probehaltig ist, widerlegt. Ebenso werden die Hilfskonstruktionen von einem „Bewusstsein uberhaupt" gegenstandslos and die nächtliche Ungehenerlichkeit, dass das Subjekt als Quell der apriorischen Formen die ganze Gesetzlichkeit in die Natur hereinlegt, nachher aber, wenn es sich gegenständlich betrachtet, als kleiner Teil dieser gesetzlichen Natur erscheint, löst sich in verständlichster Weise im Tageslicht auf.

Das Ding an sich schrumpft also damit in den wirklichen Gegenstand der Erfahrang zusammen, wie Scrooges Phantom in den Bettpfosten. Was wir apriori von den Dingen aussagen können, gilt für die Dinge selbst, nicht bloss für Vorstellungen von Dingen. Nan erst ist man in der That berechtigt, Cohens Urteil za fällen, das „Ding an sich" bezeichne nichts, als den Inbegriff aller Erkenntnisse, bezw. die unendliche Aufgabe der Erkenntnis. Es ist jetzt nicht mehr das $x$ eines fragwürdigen Rätsels, sondern in der That das $x$ einer unendlichen Gleichung, die wir in immer weiter schreitender Forschang zu lösen haben. Wenn dem so ist, so sollte man, nachdem das Gespenst des Dinges an sich vom Zauber erlöst ist anch den Namen nicht mehr im Bereiche der Erkenntnis anwenden Die unendliche Aufgabe, die wir theoretisch wie praktisch haben, bedarf beider nicht.

1) Die Thatsachen in der Wahrnehmung S. $34 \mathrm{f}$. 